\title{
Dynamic Analysis of the Fuselage of a Four-Rotor Unmanned Aerial Vehicle
}

\author{
Zhou Hongchao, Qi Yuming, Lin Weimin, Wang Peng, Xie Kunpeng \\ Tianjin University of Technology and Education \\ Institute of robotics and intelligent equipment \\ Tianjin, China
}

\begin{abstract}
The four-rotor unmanned aerial vehicle has a light and simple structure, convenient control and broad application prospects, so it has great research significance. In order to study the vibration and response characteristics of the four-rotor unmanned aerial vehicle fuselage, the Ansys Workbench was used to analyze the fuselage. First, the fuselage structure is modeled and modal analysis is performed. The results show that the excitation frequency of the four-rotor unmanned aerial vehicle avoids the natural frequency of the fuselage and avoids the occurrence of resonance. Based on the modal analysis, the harmonic response analysis is carried out. The results show that the fuselage can overcome the harmful vibration caused by the cyclic load generated by the rotor, which provides a theoretical reference and basis for the design and improvement of the fourrotor unmanned aerial vehicle.
\end{abstract}

Keywords-Four-Rotor Unmanned Aerial Vehicle; Fuselage; Dynamics Analysis; Finite Element Method

\section{INTRODUCTION}

Because of its small size and good maneuverability, the four-rotor unmanned aerial vehicle has many application scenarios and good adaptability. However, there are also deficiencies. Therefore, it has become a research hotspot in recent years. The research content is focused on flight control and flight control algorithms. There are few studies on mechanical structures and less research on dynamics. The important position of structure in machinery is self-evident. All structures are faced with dynamic problems. The dynamic characteristics of structures have become the key indicators of equipment performance and quality. Therefore, dynamic analysis of structures is an indispensable part of design and manufacture ${ }^{[1-2]}$. When the four-rotor unmanned aerial vehicle is flying, the periodic rotation of the rotor will generate periodic loads, and the load will be transmitted to the fuselage to cause forced vibration of the fuselage. If the natural frequency of the fuselage structure is similar to the rotor excitation frequency or phase at the same time, resonance will occur. Resonance will accelerate the fatigue damage of a certain part of the fuselage and reduce the service life of the drone. Therefore, it is very important to avoid resonance of the fuselage. In this paper, the modal analysis of the four-rotor unmanned aerial vehicle fuselage is carried out, and the natural frequency of the fuselage is obtained. It is calculated that the rotor excitation frequency is different from the natural frequency of the fuselage, and the two will not resonate. Based on the modal analysis. The harmonic response analysis is

This paper is supported by

the National Key Technology R\&D Program(2015BAK06B04);

the key technologies R\&D program of Tianiin(14ZCZDSF00022). carried out to verify that the fuselage can overcome the harmful vibration caused by the cyclic load generated by the rotor, which provides a reference for the design of the four-rotor unmanned aerial vehicle.

\section{PRINCIPLE OF FUSELAGE DYNAMICS ANALYSIS}

The most critical component of the four-rotor unmanned aerial vehicle is the rotor. The vibration of the rotor will cause the unmanned aerial vehicle body to vibrate, affecting the unmanned aerial vehicle's working accuracy, flight safety and service life. The four-rotor unmanned aerial vehicle has six degrees of freedom and can be regarded as a multi-degree-offreedom system. The general expression of the differential equation for a multi-degree-of-freedom linear vibration system is:

$$
M \ddot{q}+C \dot{q}+K q=Q(t)
$$

Where $M, C$ and $K$ are the $n \times n$ order mass, damping and stiffness matrices, respectively, $q, \dot{q}, \ddot{q}$ and $Q$ are the $n$ dimensional vectors of generalized coordinates, generalized velocity and generalized force, respectively.

For undamped free vibration, the above equation can be simplified as:

$$
\mathrm{M} \ddot{q}+K q=0
$$

It represents a set of $\mathrm{n}$ simultaneous homogeneous differential equations:

$$
\sum_{j=1}^{n} m_{i j} \ddot{q}_{j}+\sum_{j=1}^{n} k_{i j} q_{j}=0 \quad(\mathrm{i}, \mathrm{j}=1,2 \cdots, \mathrm{n})
$$

In motion, all coordinates have the same dependence on time. Except for the amplitude, the shape of the motion does not change, ie the ratio of the mass displacements remains unchanged. This type of exercise can be expressed as:

$$
q_{j}(t)=u_{j} f(t) \quad(\mathrm{i}, \mathrm{j}=1,2 \cdots, \mathrm{n})
$$

Where $u_{j}$ is a set of constants and $q_{j}(t)$ is all coordinates.

Acceptable solutions are:

$$
f(t)=C \sin (\omega t+\varphi)
$$

$\omega$ is the frequency of the simple harmonic motion, $C$ is an arbitrary constant, and $\varphi$ is the phase angle 


$$
\left(K-\omega^{2} M\right) u=0
$$

The condition for the existence of a non-zero solution is: if and only if the coefficient determinant is equal to zero, ie

$$
\Delta\left(\omega^{2}\right)=\operatorname{det}\left(K-\omega^{2} M\right)=0
$$

The eigenvalue is the $\mathrm{n}$ roots $\omega_{r}^{2}(r=1,2, \cdots, n)$ and their square root of the equation, $\omega_{r}(r=1,2, \cdots, n)$ is the natural frequency of the system, which is arranged from small to large. It is $\omega_{1} \leq \omega_{2} \leq \cdots \leq \omega_{r} \leq \cdots \leq \omega_{n}$, which are called first order, second order, $\cdots$, rth order, $\cdots$, and nth natural frequency, respectively. Substituting the natural frequency $\omega_{r}$ into equation (6) solves the eigenvector $u^{(r)}$, which is called a mode vector or a modal vector and is expressed as a natural mode.

\section{THE ESTABLISHMENT OF THE FUSELAGE DYNAMICS SIMULATION MODEL}

\section{A. $3 D$ model of the fuselage}

The four-rotor unmanned aerial vehicle has a wheelbase of $450 \mathrm{~mm}$ and a total weight of $1500 \mathrm{~g}$. The three-dimensional model of the four-rotor unmanned aerial vehicle fuselage structure is shown in Fig. 1. The fuselage structure is mainly the center plate and the arm. The material properties are shown in Table 1.

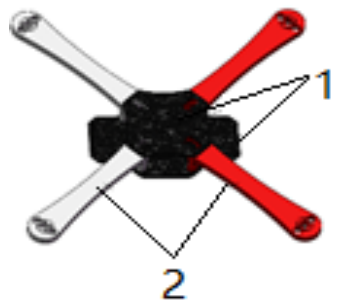

1. Center board; 2. Arm

Fig.1. Four-rotor unmanned aerial vehicle fuselage structure model

TABLEI. FOUR-ROTOR UNMANNED AERIAL VEHICLE FUSELAGE MATERIAL PROPERTY SHEET

\begin{tabular}{|c|c|c|c|c|c|}
\hline $\begin{array}{c}\text { Struct- } \\
\text { ure } \\
\text { name }\end{array}$ & $\begin{array}{c}\text { Material } \\
\text { name }\end{array}$ & $\begin{array}{c}\text { Elastic } \\
\text { Modulus } \\
(\mathrm{MPa})\end{array}$ & $\begin{array}{c}\text { Poisson's } \\
\text { ratio }\end{array}$ & $\begin{array}{c}\text { Densit- } \\
\mathrm{y}\end{array}$ & $\begin{array}{c}\text { Failure } \\
\text { stress } \\
(\mathrm{MPa})\end{array}$ \\
\hline $\begin{array}{c}\text { Center } \\
\text { board }\end{array}$ & $\begin{array}{c}\mathrm{T} 700-12 \mathrm{~K} \\
\text { carbon fiber }\end{array}$ & $2.1 \mathrm{E} 10^{\wedge} 5$ & 0.307 & 1.76 & 3400 \\
\hline Arm & $\begin{array}{c}\text { ABS } \\
\text { engineering } \\
\text { plastics }\end{array}$ & 2410 & 0.3897 & 1.07 & 90 \\
\hline
\end{tabular}

Fuselage finite element model

After setting the material properties of the fuselage in the simulation software, the contact zone of the fuselage structure is defined, and the contact type is bonded, and the mesh is divided. As shown in Fig. 2, the boundary condition is free, and no constraint is imposed on the fuselage.

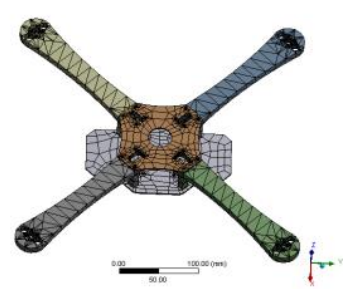

Fig.2. fuselage finite element mesh model

\section{FUSELAGE DYNAMICS SIMULATION ANALYSIS}

After the text edit has been completed, the paper is ready for the template. Duplicate the template file by using the Save As command, and use the naming convention prescribed by your conference for the name of your paper. In this newly created file, highlight all of the contents and import your prepared text file. You are now ready to style your paper; use the scroll down window on the left of the MS Word Formatting toolbar.

\section{A. Modal analysis}

The finite element software is used to analyze the four-rotor unmanned aerial vehicle fuselage with free modal analysis, which can solve the natural frequency and vibration pattern of the fuselage. Since the fuselage is in a free state without any constraints, the 1 6 order modes of the fuselage are rigid body modes, and the natural frequency is approximately 0 . Therefore, it is meaningful to start from the 7 th order mode. This paper mainly studies 7 12 order modes ${ }^{[3]}$.

TABLEII. 7 12 ORDER FUSELAGE NATURAL FREQUENCY

\begin{tabular}{|c|c|c|c|c|c|c|}
\hline Modal order & 7 & 8 & 9 & 10 & 11 & 12 \\
\hline $\begin{array}{c}\text { Natural } \\
\text { frequency }\end{array}$ & 65.398 & 83.027 & 152.54 & 161.28 & 173.48 & 194.39 \\
\hline
\end{tabular}

It can be seen from Table 2 that the natural frequency of the $7 \sim 12$ order modes, the natural frequency of the fuselage increases with the increase of the mode order, and the natural frequencies of the 7 th and 8 th orders are similar. The natural frequencies of the 9th to 12th order are similar, but the frequency difference between the 11th and 12th orders is larger than the 9th and 10th, 10th and 11th orders.

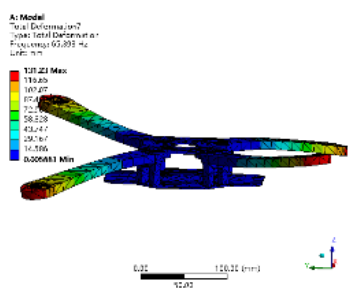

7 th order mode shape

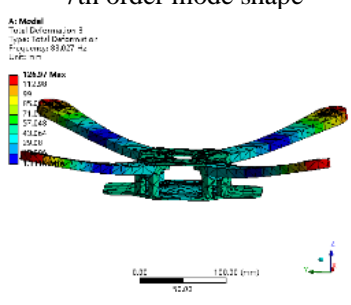

8th order mode shape 


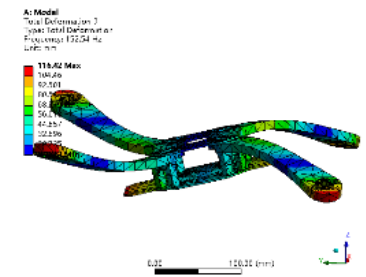

9th order mode shape

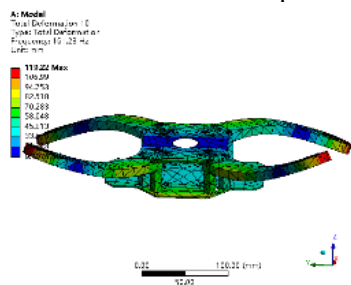

10th order mode shape

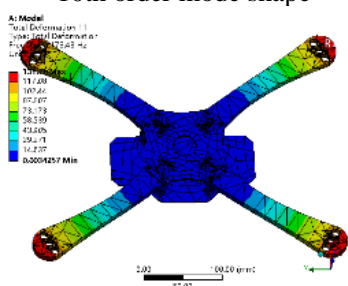

11 th order mode shape

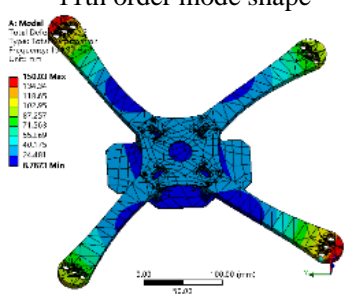

12th order mode shape

Fig.3. 7 12th order modes shape cloud image

It can be seen from Fig. 3 that the 7 th order model shape is the torsional deformation of the arm, the 8th order model shape is the bending deformation of the arm, the 9th order model shape is the overall torsional deformation of the fuselage in the $\mathrm{X}$ direction, and the 10th order model shape is the overall torsional deformation of the fuselage in the $\mathrm{Y}$ direction. The 11th order model shape is that the arm is symmetrically bent and deformed along the $\mathrm{Z}$ plane of the fuselage, and the 12th order model shape is that the fuselage is bent and deformed along the positive direction of the $\mathrm{Y}$ axis. If the fuselage vibrates in a certain mode order of 7 12 order model, the corresponding vibration deformation will occur in the fuselage or a part, and most of the deformation will occur at the arm of the fuselage.

The four-rotor unmanned aerial vehicle adopts the KV980 motor. The rotation speed is $10878 \mathrm{r} / \mathrm{min}$ at a voltage of $11.1 \mathrm{~V}$. This is the fastest design speed. The rotor excitation frequency can be calculated by the formula $\omega=z p$ and the excitation frequency is $362.6 \mathrm{~Hz}$. Where $z$ is the number of rotor blades and $p$ is the rotor speed. It can be seen from Fig. 4 that the rotor

\section{CONCLUSION}

In this paper, the dynamic analysis of the fuselage structure of a four-rotor unmanned aerial vehicle is carried out, and the following conclusions are drawn. excitation frequency is between the 13th and 14th natural frequencies and does not resonate with the fuselage structure ${ }^{[4]}$.

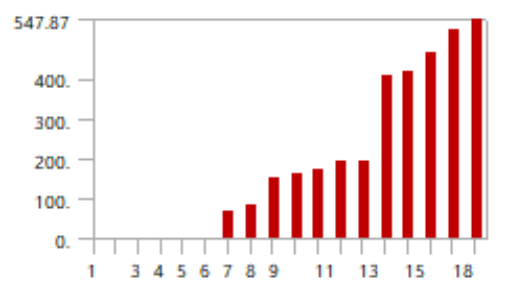

Fig.4. 1 18 order fuselage natural frequency

\section{B. Harmonic response analysis}

In order to understand the influence of the rotor on the vibration of the fuselage in more detail, the harmonic response analysis is carried out on the basis of the modal analysis, and the fuselage is restrained ${ }^{[5-6]}$. The $\mathrm{Z}$ positive direction is set to $12 \mathrm{~N}$, and the frequency range is $0 \sim 200 \mathrm{~Hz}$. The solution interval is 4 . It can be seen from Fig. 5 and Fig. 6 that when the harmonic response frequency of the fuselage is about $65 \mathrm{~Hz}$, approached the 7 th order natural frequency of the fuselage, the equivalent stress and deformation of the fuselage are maximized, and the center of the fuselage is bent and deformed in the negative direction of the Z-axis. The position of the maximum value appears at the center of the fuselage. The central plate has the maximum stress. The maximum equivalent stress is $95.42 \mathrm{MPa}$, and the maximum deformation is $20.37 \mathrm{~mm}$. When the harmonic response frequency of the fuselage is about $175 \mathrm{~Hz}$, it is close to the 11th order natural frequency of the fuselage. The maximum equivalent stress is $1.78 \mathrm{MPa}$, and the maximum deformation is $0.19 \mathrm{~mm}$, which is much smaller than the failure strength of the fuselage material. The strength and rigidity of the fuselage meet the design and use requirements. It is verified that the fuselage can overcome the harmful vibration caused by the cyclic load generated by the rotor.

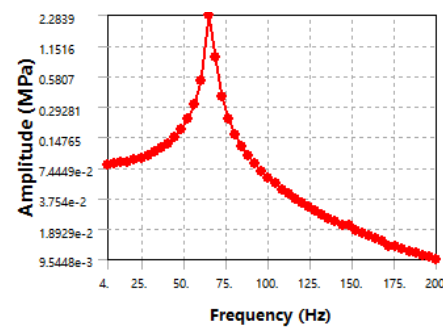

Fig.5. Fuselage stress frequency response

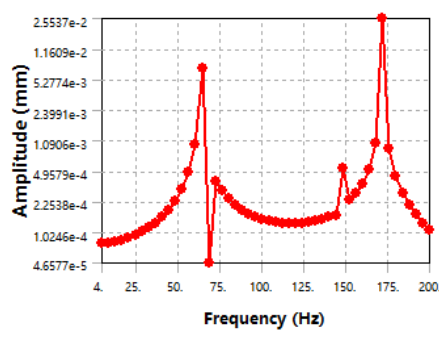

Fig.6. Fuselage deformation frequency response

- Through the modal analysis of the fuselage, the simulation results show that the rotor excitation frequency completely avoids the natural frequency of the fuselage structure and avoids the resonance. 
- Through the harmonic response analysis, it is verified that the fuselage structure can overcome the forced vibration caused by the wind.

- After dynamic analysis, it is found that the stress and deformation values at the center of the fuselage are the largest, and the center plate can be appropriately thickened for optimization.

\section{REFERENCES}

[1] LI Zhan-ke, SONG Bi-feng, SONG Hai-long. Study on Actualities of Micro Air Vehicles and Its Key Technologies [J]. Flight Dynamics, 2003, 21 (4):1-4.

[2] Stephen J. Mc Gilvray. Attitude stabilization ofa Quadrotor aircraft [D],2004.

[3] ZHANG Zhi-long, YIN Ming-de, LIU Jin-jin. Modal Analysis of Light Helicopter Fuselage [J]. Mechanical Engineering \& Automation, 2013 (01) :35-37.

[4] WEI Wen-fei, ZHANG Chun-yuan, LI Chao, et al. Modal Analysis of Four Rotor Aircraft Frame[J]. Journal of Ordnance Equipment Engineering,2017 (2) :40-42.

[5] Yang Yong, Wang Shilong, Tian Zhifeng. Dynamics Simulation Analysis of Column for Large-scale NC Gear Hobbing Machines [J]. China Mechanical Engineering,2013,24 (11) :1-6.

[6] XIONG Yonghua, DU Farong, GAO Feng, et al. Research and Analysis on dynamic characteristics of light truck [J].Journal of Machine Design,2007,24 (4) :60-62. 